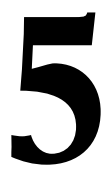

\title{
AMISTADES CO-ÉTNICAS E INTER-ÉTNICAS EN LA ADOLESCENCIA: DIFERENCIAS EN CALIDAD, CONFLICTO Y RESOLUCIÓN DE PROBLEMAS
}

\author{
(CO-ETHNIC AND INTER-ETHNIC FRIENDSHIPS IN ADOLESCENCE: \\ DIFFERENCES IN QUALITY, CONFLICT, AND PROBLEM SOLVING)
}

Cecilia Francisca Villalobos Carrasco

Universidad de Barcelona

Ibis Marlene Álvarez Valdivia

Universitat Autònoma de Barcelona

Elizabeth Vaquera

University of South Florida, EE.UU.

DOI: 10.5944/educXX1.17493

\section{Cómo referenciar este artículo/How to reference this article:}

Villalobos Carrasco, C.F.; Álvarez Valdivia, I.M., y Vaquera, E. (2017). Amistades co-étnicas e inter-étnicas en la adolescencia: Diferencias en calidad, conflicto y resolución de problemas. Educación XX1, 20(1), 99-120, doi: 10.5944/educXX1.17493

Villalobos Carrasco, C.F.; Álvarez Valdivia, I.M. \& Vaquera, E. (2017). Amistades co-étnicas e inter-étnicas en la adolescencia: diferencias en calidad, conflicto y resolución de problemas. [Coethnic and inter-ethnic friendships in adolescence: Differences in quality, conflict, and problem solving]. Educación XX1, 20(1), 99-120, doi: 10.5944/educXX1.17493

\section{RESUMEN}

En los últimos años, la llegada masiva de población extranjera en edad escolar a Cataluña ha incrementado la diversidad cultural en las aulas. En este contexto, los amigos son un soporte importante que contribuye a una mejor integración. El objetivo de este estudio fue identificar y comparar los valores que definen la calidad, el conflicto y sus formas de resolución en amistades co-étnicas e inter-étnicas de adolescentes en Institutos de Educación Secundaria Obligatoria, con presencia significativa de estudiantes de origen extranjero. Para ello se realizó una entrevista semiestructurada. Participaron 90 estudiantes correspondientes a 45 diadas; 20 diadas de amigos co-étnicos y 25 de amigos inter-étnicos. Los resultados evidencian que para diadas co-étnicas los valores más importantes son la confianza y la compañía y para diadas inter-étnicas la confianza y la ayuda. Los amigos de ambos tipos de diadas 
refieren que los conflictos ocurren por desacuerdos generando sentimientos de enfado o tristeza. Adicionalmente, las diadas inter-étnicas destacan que la falta de lealtad es un motivo que pone en peligro la amistad. Ambos tipos de diadas utilizan estrategias similares en la resolución de sus diferencias, primero intentan hablar de lo sucedido para llegar a un acuerdo y esto en general les trae sensaciones de alivio, se sienten reconfortados por mantener su relación intacta y poder continuar disfrutando de la compañía de su amigo, aumentando la tranquilidad y el bienestar personal. Estos resultados dejan al descubierto que es necesario promover espacios para la integración que permitan generar relaciones de amistad diádica en contextos educativos de diversidad cultural.

\section{PALABRAS CLAVE}

Amistad; adolescentes; relaciones entre iguales; comunicación intercultural; conflicto; educación secundaria; inmigrantes.

\section{ABSTRACT}

In recent years, the mass arrival of foreign population of school age in Catalonia has increased cultural diversity in the classroom. In this context, friends are an important support that contributes to better integration. The aim of this study was to describe and compare inter-ethnic and co-ethnic friendship relations in terms of their friendship quality, conflict, and problem solving strategies. The sample is drawn from secondary school students in schools with significant presence of immigrants. Ninety students in 45 friendship dyads participated in a semi-structured interview. In total, the sample includes 20 dyads of co-ethnic friendships and 25 dyads of inter-ethnic friendships. Results show that for co-ethnic dyads the most important friendship values are trust and companionship. Among inter-ethnic friendships it is trust and support. In co-ethnic dyads conflict occurs due to disagreements that result in anger or sadness. Inter-ethnic dyads are similar in their reports of conflict, but they also emphasize lack of loyalty as a reason of conflict. Both types of dyads use similar strategies for conflict resolution: to talk about what happened, which brings them a sense of relief and comfort, which in turn increases ease and wellbeing. These results unveil the need to promote spaces for integration that can generate dyadic friendship relations in educational contexts of cultural diversity.

\section{KEYWORDS}

Friendship; adolescents; peer relationship; intercultural communication; conflict; secondary education; immigrants. 


\section{INTRODUCCIÓN}

En la última década, España ha experimentado un aumento de población extranjera; específicamente Cataluña ha sido el mayor receptor de inmigrantes con un 15,8\% de la población total (INE, 2012) y es posible apreciar diversas culturas, lenguas e identidades presentes en la población. El contexto escolar no ha estado ausente de este proceso; la presencia de un número importante de alumnos inmigrantes ha provocado transformaciones del sistema educativo, entre ellas la incorporación de la acogida personalizada y atención lingüística, dado que un 13,7\% de los alumnos son inmigrantes (Generalitat de Catalunya, 2012). Los adolescentes, al convivir con distintas culturas, deben hacer adaptaciones en la configuración de su identidad para aceptar, y muchas veces tolerar, distintas costumbres, idiomas, etnias, y religiones, lo cual moldea su personalidad (Azmitia, Ittel y Radmacher, 2005). En este contexto los amigos son un soporte importante que funciona como un sistema de relaciones que surge de la interacción social con el grupo de iguales (Giró, 2011) favoreciendo la socialización.

Considerando la elevada presencia de adolescentes de origen extranjero en Cataluña, en esta investigación describimos amistades que se establecen en un contexto educativo multicultural. Usando un protocolo de entrevista, nos interesa identificar y comparar las diadas de amigos coétnicos e inter-étnicos respecto a los valores que definen la calidad de las amistades, los conflictos y formas de superar los conflictos. Argumentamos que las amistades son un recurso importante que favorece la integración de adolescentes en la escuela. En el caso de estudiantes de origen extranjero esto sería un factor protector para una mejor integración social y educativa. Además, pensando en el aumento gradual y significativo de diferentes culturas que se establecen en el país, es de suma importancia el conocer cómo se comportan los adolescentes en una sociedad que no es propia para así poder implementar mejoras en el sistema educativo que ayuden a fortalecer las relaciones de amistad de las distintas culturas y favorezca el acceso de información del nuevo contexto en el que se incorporan.

\section{Amistad adolescente en contextos interculturales}

La amistad se caracteriza por ser un vínculo afectivo, construido entre dos personas que cuando están juntas es fuente de sentimientos de placer, apoyo emocional y de satisfacción personal. Más concretamente, este vínculo ha sido definido como una relación diádica (entre dos personas) caracterizada por intimidad mutua, compañía, apoyo y capacidad para resolver problemas en momentos difíciles (Bukowski y Hoza, 1989; Schneider, Dixon y Udvari, 2007; Schneider, Malik y Udvari, 2012). Un rasgo distintivo 

AMISTADES CO-ÉTNICAS E INTER-ÉTNICAS EN LA ADOLESCENCIA: DIFERENCIAS...

básico de la amistad es su reciprocidad y es una de las expectativas de las relaciones afectivas. Cuando una persona piensa en otra como «mi amigo/a» lleva implícito que la otra persona también piensa en él/ella como su amigo (Vaquera y Kao, 2008).

Los centros educativos tienen un importante papel en el desarrollo social, emocional e intelectual de sus estudiantes, dado que es ahí donde los adolescentes pasan la mayor parte del tiempo y por ende donde pueden generar espacios de convivencia propicios para que se establezcan relaciones de amistad. Durante la adolescencia los amigos son capaces de reflexionar sobre su conducta y la de otros, sobre sus emociones e intenciones, generando nuevas ideas sobre la amistad que se reflejan en la interpretación de sus experiencias (Azmitia et al., 2005; Newman, Erdley y Marshall, 2011).

La mayoría de estudios sobre la calidad, estabilidad y naturaleza de amistades que se generan entre adolescentes de una misma cultura (amistades co-étnicas) y entre adolescentes procedentes de diferentes culturas (amistades inter-étnicas) se han realizado en Norte América, Europa (Hallinan y Williams, 1989; McGlothlin y Killen, 2006; Schneider et al., 2007) y más recientemente en España (Alarcón, 2010; Álvarez, Hernández y Vall, 2013; Aparicio, Portes y Haller, 2009; De Miguel y Carvajal, 2007; Serra, 2006). En general, estos estudios muestran un alto grado de encapsulación, es decir, tienden a ser amigos de otros adolescentes de su misma cultura/ etnia/raza, pero todas las investigaciones tienden a apoyar que las amistades inter-étnicas tienen efectos positivos en los adolescentes (Feddes, Noack y Rutland, 2009; Munniksma y Juvonen, 2012; Pica-Smith, 2009). En este estudio partimos del supuesto que mantener amistades de calidad favorece el bienestar y facilita la integración social y educativa de los adolescentes de origen extranjero, especialmente en contextos de alta diversidad cultural, como es el caso de Cataluña.

\section{Calidad de las amistades}

En la adolescencia, la amistad es percibida como una relación continua, los amigos son personas con las que se puede contar, se comprenden y se dan apoyo social (Rubin, Bukowski y Parker, 2006). En esta etapa las amistades pasan a tomar un papel primordial porque los jóvenes sienten la necesidad de incrementar el número de relaciones que tienen con sus compañeros y estos vínculos se tornan cada vez más íntimos y significativos. Establecer este tipo de vinculación tiene dos funciones: por un lado los amigos pueden servir como fuente de apoyo o admiración y por el otro les permite compartir experiencias con otras personas que tienen los mismos problemas que ellos y una posición semejante en el mundo (Buhrmester, 1990). 

AMISTADES CO-ÉTNICAS E INTER-ÉTNICAS EN LA ADOLESCENCIA: DIFERENCIAS...

Tener amistades de calidad con las que se mantiene una relación cercana y positiva, mejora la autoestima, la competencia social y bienestar psicológico; así como también ayuda a desarrollar la personalidad y el crecimiento socioemocional (Álvarez, Schneider y Villalobos, 2015; Bukowski, 2004; Sharabany y Schneider, 2004), cuestiones esenciales para el fortalecimiento de la identidad en la adolescencia. Si los amigos se han elegido recíprocamente, es probable que perciban la relación como de calidad, con menos conflictos de cómo lo verían dos adolescentes que no se nominan el uno al otro (Linden-Andersen, Markiewicz y Doyle, 2008).

En específico, las relaciones de calidad —en términos de apoyo, intimidad y reciprocidad-con amigos y compañeros suelen fomentar el mantenimiento de los vínculos y pueden contribuir a un mejor ajuste psicosocial (Bagwell y Schmidt, 2011). Desde este punto de vista, la amistad parece ser la experiencia relacional que, a través del apoyo y la comparación social, ofrece a los adolescentes la oportunidad para aprender a regular las emociones y para desarrollar o fortalecer sus competencias sociales (Chong., Holly y Buhrmester, 2013) y académicas (Vaquera y Kao, 2012; Wentzel, McNamara y Caldwell, 2004).

Una de las funciones más importantes de la amistad adolescente es ofrecer un espacio de placer y seguridad fuera de la familia, donde pueden explorar el efecto de sus comportamientos sobre ellos mismos, sus compañeros y el entorno (Rubin et al., 2008). Atribuyen gran valor a aspectos psicológicos y toma importancia el papel que tiene la amistad en la satisfacción de necesidades emocionales como la ayuda mutua, intimidad, autenticidad, confianza, reciprocidad, lealtad, compañía, afectividad, aceptación mutua $\mathrm{y}$ al hecho de tener gustos y aspiraciones afines (Bukowski, Hoza y Boivin, 1994) por lo que escogen con mayor frecuencia a amigos que comparten sus mismas conductas, actitudes, intereses e identidades.

Por otra parte, las investigaciones que se han centrado en estudiar qué sucede con la calidad de amistades interculturales coinciden en señalar que las amistades inter-étnicas son menos estables que las co-étnicas. Por ejemplo, en un estudio realizado en dos escuelas de secundaria de Toronto con presencia significativa de inmigrantes, Smith y Schneider (2000) encontraron que los adolescentes en su mayoría tienen amistades de diverso origen étnico, existiendo variación en la calidad de las amistades co-étnicas e inter-étnicas. Los participantes de Asia oriental calificaron como de mayor calidad sus amistades con miembros del propio grupo (amistades co-étnicas) que las que tenían con miembros de otros grupos étnicos. Esto no ocurrió para los grupos de origen indio ni para los anglo-europeos. Por otro lado, el estudio longitudinal llevado a cabo por Schneider et al. (2007) con adolescentes de escuelas en barrios mul- 
ticulturales de Toronto y Montreal, también evidenció que las amistades co-étnicas son más estables, tienen mejor calidad y se caracterizan por ser más cercanas y menos competitivas que las amistades inter-étnicas, a pesar de que son tendentes a aumentar los conflictos con el tiempo. En España, concretamente en Cataluña, también se ha comprobado que las amistades co-étnicas son más estables que las amistades inter-étnicas, las cuales se caracterizan por presentar mayor competencia y menos intimidad (Serra, 2006).

\section{Conflicto en las amistades}

Las relaciones de amistad de los adolescentes también pueden pasar por momentos de dificultad especialmente cuando se confrontan opiniones o intereses. No obstante, en una amistad de calidad buscar solución al conflicto es un ideal y una meta importante ya que esta circunstancia puede poner en peligro la relación causando su deterioro o disolución; incluso, es muy probable que los amigos eviten enfrentamientos por temor o miedo a perder la amistad (Azmitia et al., 2005). Si la amistad tiene un buen nivel de calidad, la mayoría de los conflictos se soluciona de forma amistosa poniendo en práctica estrategias de mitigación para minimizar los problemas y asumiendo el reto que supone la gestión eficiente de conflictos interpersonales (Burk y Laursen, 2005). Por el contrario, cuando los jóvenes perciben la relación como menos conflictiva, lo asocian con una amistad de menor calidad (Demir y Urberg, 2004). En cualquier caso, los adolescentes que tienen más competencias en el manejo de los conflictos (por ejemplo: mayor empatía) tienden a tener menos discrepancia en sus amistades (Chong, et al., 2013).

\section{MÉTODO}

\section{Participantes}

Para esta investigación utilizamos datos de un estudio realizado entre año 2010-2011 (Álvarez, et al., 2013) en seis institutos de Barcelona y Girona con alto porcentaje de estudiantes de origen extranjero (alrededor del 33\%). Según el origen étnico de los estudiantes extranjeros en estos institutos, un $61 \%$ provenientes de Latinoamérica; un 13\% de Medio Oriente; Un 12\% de Europa; un 10\% de Asia y un 4\% de África. En concreto, los resultados de este estudio están basados en los relatos de 90 entrevistas en profundidad, o 45 diadas. Según la composición étnica, se entrevistaron a 20 diadas de amigos co-étnicos (ambos estudiantes de una misma cultura) y 25 diadas de amigos inter-étnicos (adolescentes de culturas diferentes). Por género, 
21 diadas estaban compuestas por mujeres, 20 por hombres y 4 diadas eran mixtas. Los jóvenes entrevistados cursaban el $3 .^{\circ} \mathrm{o}$ el $4 .^{\circ}$ curso de Educación Secundaria Obligatoria, con un promedio de edad de 14,8 años. Estas diadas fueron propuestas por los profesores tutores de $3 .^{\circ}$ y $4 .^{\circ}$ de ESO. Se les pidió que completaran una ficha con las parejas de mejores amigos existentes en su curso, privilegiando mencionar parejas de amigos conformadas por estudiantes de origen extranjero.

\section{Instrumentos}

Entrevista semiestructurada en profundidad: (protocolo adaptado de Azmitia et al., 2005). A través de esta entrevista se procura que los adolescentes narren la historia de su amistad, con lo cual a la vez tienen la posibilidad de identificar sucesos importantes de su relación de amistad, indagar sobre la filosofía (valores) y sobre conflictos vividos. A cada uno de los estudiantes integrantes de las diadas seleccionadas se les preguntó separadamente acerca de sus filosofías sobre la amistad y sobre los conflictos con su amigo/a. En primer lugar se les invitó a relatar sus ideales, valores y expectativas de una amistad estrecha, semejanzas y diferencias con sus amigos y/o amigas. Seguidamente se indagó sobre los motivos de conflicto y sobre los sentimientos experimentados durante y después de estos. Además se sondearon los modos de afrontarlo, preguntando si hicieron algo para solucionarlo. Las preguntas finales permitieron explorar la historia de su amistad por ejemplo, cambios producidos en la amistad a través del tiempo. La entrevista estaba compuesta por un total de quince preguntas abiertas y tuvo una duración aproximada de 15 minutos.

\section{Procedimiento}

Para llevar a cabo este estudio se contó con el consentimiento de los centros educativos y también de los alumnos y sus profesores tutores. Las entrevistas aplicadas a los 90 adolescentes se realizaron en horario lectivo, en una sala tranquila facilitada para dicha tarea. En las entrevistas se introdujeron algunos de los indicadores utilizados en el cuestionario de evaluación de la amistad de Bukowski, et al. (1994) para indagar en específico sobre su calidad, los conflictos y sus motivos. Las entrevistadoras fueron entrenadas para realizar primero un conjunto estándar de preguntas y para, adicionalmente de ser necesario, hacer preguntas complementarias que permitieran ajustar las respuestas de los participantes a los propósitos de la entrevista y obtener así la mayor cantidad de información respecto a sus experiencias de amistad. 

AMISTADES CO-ÉTNICAS E INTER-ÉTNICAS EN LA ADOLESCENCIA: DIFERENCIAS...

Las entrevistas se grabaron en audio y se transcribieron. Para el análisis inicial las respuestas fueron asignadas a tres temas:

Calidad de la amistad: Relacionado a las filosofías de la amistad, incluyendo valores específicos tales como confianza, compañía, respeto, ayuda, diversión, escucha y afecto mutuo.

Conflicto: Conformada por motivos que ponen en peligro la amistad (falta de lealtad, indiferencia, bromas pesadas). En este tema también se incluyen los relatos referidos a reacciones emocionales desagradables asociadas a experiencias conflictivas, como sentimientos de soledad, enfado y/o tristeza.

Trascender el conflicto: Referido a las estrategias utilizadas para resolver el conflicto (negociación y/o distanciamiento) y a las vivencias o sentimientos que les provocaba el solucionarlo (alivio y/o rencor).

Posteriormente, para un análisis más exhaustivo, a cada tema se le asignaron categorías emergentes que fueron validadas de manera independiente por tres jueces investigadores con experiencia en el tema de las amistades. En este proceso de las 58 categorías iniciales, se validaron 39 (86\% de acuerdo interjueces), que fueron clasificadas en sus respectivos temas y subtemas. Finalmente, los 90 relatos que surgen de las entrevistas fueron analizados en base a estas categorías.

\section{RESULTADOS}

Los resultados se darán separadamente para los temas de calidad, conflicto y sus formas de resolución tanto para diadas co-étnicas como inter-étnicas, mostrando los extractos de los relatos más representativos. Partimos de la base de que cuando en una relación de amistad los jóvenes se sienten cómodos, pueden expresarse libremente, como lo evidencia el relato de un chico de 16 años perteneciente a una diada co-étnica al hablar de la amistad: «En una amistad puedes hacer todo lo que quieras, pasártelo bien, mal, llorar, reír, expresarte de la forma que quieras... siempre con total sinceridad", la seguridad que le da el otro para hablar y contar sus cosas es tal, que lo expresa sin necesidad de modificar su forma para caer bien al otro. Lo que pretendemos es mostrar la riqueza de sus relatos en los resultados.

\section{Amistades Co-étnicas e Inter-étnicas ¿diferencias en la calidad?}

Cuando les preguntamos por la calidad de su amistad, indagamos respecto a cuáles son los valores y expectativas que ellos tienen de una amistad cercana. En general, tanto en diadas co-étnicas como en inter-étnicas el valor 

AMISTADES CO-ÉTNICAS E INTER-ÉTNICAS EN LA ADOLESCENCIA: DIFERENCIAS...

más importante dentro de una amistad es la confianza, entendida como la seguridad que le da el otro para hablar y contar sus cosas libremente, para guardar secretos o aconsejar. Sin embargo, se observaron diferencias en el segundo valor mencionado; los adolescentes de diadas co-étnicas mencionan que para ellos es importante la compañía, el tener la presencia física del amigo, el que sea buena persona y puedan compartir cosas en común, en cambio las diadas inter-étnicas atribuyen consideración especial a la ayuda, la colaboración en momentos difíciles, al apoyo incondicional que le da el amigo, que sea solidario y lo aconseje si es necesario, como se podrá apreciar en sus relatos.

\section{Diadas Co-étnicas: Confianza y Compañia}

Cuando se les preguntó cuáles eran los valores que esperaban de una buena amistad, Alberto (16) un adolescente español nos dijo: «Para mílo más importante es total confianza, porque una amistad no es amistad si no hay un vínculo muy fuerte donde puedes expresar todo lo que quieras...». Luego, al consultarles por qué consideraban especial la amistad con este amigo, Xavier (16), catalán, expresó: «Porque tengo mucha más confianza con él, me atrevo a decir más cosas, o sea... no me cuesta hablar de cosas más personales con él, de cosas más íntimas y eso lo valoro mucho». La valoración puede llegar a tal punto que se cree que el nivel de confianza que existe es sorprendente, Dolores (14), peruana nos dice: "A veces con una mirada sabemos lo que pensamos, es fácil hablar con ella y es fácil la comunicación, es muy fácil y te entiendes enseguida», con referencia a su amiga peruana. Estos extractos nos muestran que la confianza es muy importante para los adolescentes, les permite expresarse y compartir sus miedos y alegrías de forma espontánea.

Otro valor que también mencionan con frecuencia estas diadas es la compañía. Mercè (14) una adolescente catalana relata: «Podemos hacer muchas cosas juntas, siempre nos tenemos la una a la otra», dando a conocer la importancia que otorgan a la realización de actividades con amigos cercanos, lo que muchas veces también está asociado con diversión.

En general, para las diadas co-étnicas la valoración asignada a la confianza implica un lazo profundo que va más allá del que podría tener con un amigo cualquiera y para ellos la compañía, el «sentirse acompañados», es también sinónimo de bienestar.

\section{Diadas Inter-étnicas: Confianza y ayuda}

Del mismo modo que en las amistades co-étnicas, los adolescentes en diadas inter-étnicas mencionaron que el valor más importante que podían recibir de una amistad cercana era la confianza. Ana Lucía (14), una estu- 
diante ecuatoriana que menciona como mejor amiga a una catalana, relató: "Mi mejor amiga... lo que pienso es que debe ser una persona en la cual puedo confiar y puedo contar con ella siempre y tener esa relación..., ese sabes... de poder confiar en ella en cualquier cosa». En la confianza atribuida subyace una expectativa de reciprocidad, como lo expresa Anahit (15), de Armenia, cuando nos dijo por qué considera especial la amistad con su amiga peruana: «Porque nos contamos las cosas. Sabemos todo..., o sea, sabemos todo de las dos, yo le cuento mis cosas y ella me cuenta las suyas. Es la persona a la que le cuento todo, por eso se diferencia de otras chicas» recalcando que esta reciprocidad en la confianza marca la diferencia con el resto de sus amigas.

A diferencia de las diadas co-étnicas, en diadas inter-étnicas el valor que mencionan como segunda opción en orden de importancia es la ayuda recibida por parte del amigo. Los adolescentes en sus relatos dejan entrever que para ellos es importante y necesario sentirse apoyados, Tatiana (17) de Honduras con amiga catalana menciona: «Es buena amiga porque me ayuda, porque está conmigo siempre, cuando lloro y cuando río y está siempre que la necesito... la llamo a cualquier hora, siempre está conmigo». Esta misma adolescente también valora la ayuda recibida por su amiga en su proceso de integración, "Creo que yo nunca me había integrado tan bien a la sociedad vale... ella conocía a mucha gente y tal... y pues yo también me he dado a conocer... no sé, soy conocida ivaya!', dejando en evidencia que gracias a la ayuda de su amiga, ahora se siente integrada y reconocida ante el resto de adolescentes. Podemos apreciar que cuando los adolescentes provienen de culturas diferentes, la ayuda que puede prestar un adolescente de la cultura de acogida es primordial y de suma importancia para la socialización, por lo que creemos necesario que en la escuela se promuevan este tipo de alianzas.

En diadas inter-étnicas la confianza significa poder contar cosas íntimas que no contarían a cualquier persona. Implica reciprocidad, donde ambos amigos deben tener las mismas oportunidades para expresarse. Cuando ambos amigos de la diada son de origen extranjero se sienten más unidos y comprendidos al vivenciar procesos similares y cuando la diada la componen un inmigrante y un catalán la valoración asignada a la ayuda es primordial, lo que podría significar un puente a la integración de los inmigrantes al sentirse valorados dentro de la sociedad donde están viviendo.

\section{Amistades Co-étnicas e Inter-étnicas, ¿Existen diferencias en los motivos de conflicto de las amistades y en los sentimientos que esto les provoca?}

Cuando les preguntamos por los conflictos, indagamos respecto a los motivos que ponen en peligro una amistad y cómo lo enfrentan. En general, 

AMISTADES CO-ÉTNICAS E INTER-ÉTNICAS EN LA ADOLESCENCIA: DIFERENCIAS...

ambos tipos de diadas al narrar algún conflicto/pelea con su amigo mencionaban que no existían grandes conflictos, solo pequeños discusiones/desacuerdos que podían o no afectar la amistad. En diadas co-étnicas encontramos que los conflictos ocurren por algunos desacuerdos y los solucionan con enfado o tristeza. En diadas inter-étnicas los resultados son similares, pero ellos enfatizan en la falta de lealtad como un motivo generador de conflicto, lo cual se percibe como una importante amenaza a la relación de amistad.

\section{Desacuerdos en Diadas Co-étnicas: Enfado o tristeza}

Cuando se les consultó por los conflictos que ocasionaban problemas en la relación de amistad relataron sus desacuerdos. Mar (15) adolescente catalana expresó: "Las dos somos muy tozudas y siempre regañamos, son pequeños conflictos que acabamos riendo siempre. Puede ser que una ha tenido un mal día y contesta muy mal y durante todo el día estamos distanciadas» evidenciando que no hay enojo, pero de igual forma queda con una sensación de malestar emocional.

En general, las emociones asociadas a estos conflictos son para algunos el enfado y para otros la tristeza. En ocasiones cuando los adolescentes se sienten marginados de algunas situaciones que ellos consideran importantes, está latente la sensación de enojo, como lo expresa Albert (16) de España: "Yo me sentía estafado sabes, era algo importante para ella, que la hacía feliz, para mí es importante decirle cómo te sientes», aludiendo además que el enojo hay que expresarlo. Por otro lado, ante las dificultades con el amigo, muchos adolescentes reaccionan con tristeza, se sienten afligidos o abatidos por la ruptura temporal de la amistad, lo que se hace evidente e interfiere en la forma de comportarse en el colegio o en el hogar, como lo expresa María (14) de Ecuador: "Yo estaba en mi casa y estaba pensando en la forma de hablarle, estaba pensativa...en casa me decían "¿chica qué te pasa?" y yo "no, es que mi mejor amiga...". Siempre estaba pensando...en el colegio, en casa... estaba un poco así... de bajón...».

En resumen, observamos que los motivos de conflictos en diadas co-étnicas se derivan por desacuerdos, por malentendidos que generan opiniones discordantes y que provocan «pequeñas» rencillas a las que reaccionan molestándose y enfadándose con el amigo, o bien experimentan una sensación de pena o tristeza.

\section{Desacuerdos y falta de lealtad en Diadas Inter-étnicas: Enfado o Tristeza}

Del mismo modo que en las diadas co-étnicas, en las inter-étnicas los conflictos suelen ocurrir por desacuerdos, pero además por falta de lealtad. La vivencia que se experimenta frente a estos conflictos es de enfado o tristeza. 

AMISTADES CO-ÉTNICAS E INTER-ÉTNICAS EN LA ADOLESCENCIA: DIFERENCIAS...

Cuando les preguntamos que intentaran imaginar qué situación o motivo haría perder la amistad con su amigo o bien que comentaran algún conflicto, en primer lugar se refirieron a los desacuerdos. Cuando los adolescentes describen el comportamiento del amigo, no necesariamente se refieren a situaciones que causen dificultades con ellos, sino que una conducta del amigo puede ser motivo de desacuerdos. Por ejemplo, Nasreen (15) de Pakistán nos relató: "A veces empieza a hablar de cosas que a mí no me gustan nada, comienza a criticar a otra gente y así y por eso nos peleamos», de manera que aunque no sea un problema directo con ellos, las actitudes del amigo pueden llegar a afectar la relación.

Otro tipo de situación que es motivo de desacuerdos en las amistades inter-étnicas se relaciona con dificultades para el aprendizaje del idioma, como expresa Miquel (14), adolescente catalán al referirse a su amigo ruso: "A mi amigo le enfada que lo corrijan... no sé, una palabra, una frase. Habla el castellano y el catalán bien pero hay algunas palabras que no entiende. Cuando el profesor o sus otros amigos le corrigen una palabra, algo... no sé... una frase vale, le corrigen y lo odia. Yo una vez hice eso y a él no le gustó nada», evidenciando que los desacuerdos por causa del aprendizaje del idioma tienen una sensibilidad especial para adolescentes inmigrantes, el sentirse que son objeto de burlas hiere su ego y les trae desavenencias con sus amigos y con el resto de sus compañeros.

Cuando los adolescentes de las diadas inter-étnicas relatan los motivos de conflicto que ponen en peligro la amistad, además de los desacuerdos, y a diferencia de las co-étnicas, añaden falta de lealtad (referida a experiencias que les costaría perdonar a su amigo, como hablar a sus espaldas, si es desleal, si miente, si inventa historias que los perjudiquen, rompe promesas o cuenta secretos). Agnieska (15) de Polonia ante esta pregunta contestó que las mentiras eran algo que no podría perdonar: «Mentirme y que luego yo sepa que me miente y que me continúe mintiendo, eso me da muchísima rabia, las mentiras sobre todo». También mencionan el contar secretos a otros, como lo expresa Paulo (14) de Portugal: "El que cuenten algo íntimo es motivo de perder una amistad, de hecho antes con un compañero de clase éramos muy buenos amigos, era como un hermano. Ahora no lo considero un amigo, somos muy diferentes... nuestra amistad se estropeo así». La contrariedad o crítica también es mencionada, como se ejemplifica en el relato de Román (16) de Ucrania: «Si empieza a criticarme a mis espaldas, me daría mucha rabia».

Igual que en las diadas co-étnicas, las emociones que en mayor medida involucran estas situaciones conflictivas en las diadas inter-étnicas suelen ser el enfado y la tristeza, predominando el enfado. Por ejemplo, observamos que cuando los amigos de diadas inter-étnicas se enfadan, perciben 
una sensación de enojo, desagrado y que afecta la relación, como lo expresa Cristian (16), de Chile, al hacerle una broma a su amiga: «Un día estábamos hablando de los inmigrantes, entonces yo sé de dónde provengo y cuál es mi cultura y que voy a ser chileno toda mi vida; en cambio Adela se avergüenza un poco de ser de Ecuador y no le gusta el estereotipo que se tiene de la gente de su país. Entonces estábamos hablando de esas cosas, yo hice una broma de que "Adela no era latina", y ella se enfadó muy en serio por eso. Estuvimos enfadados dos días».

Además del enfado, con frecuencia encontramos relatos de tristeza, como lo expresa Teresa (15) de Honduras: «Nos enfadamos una vez por culpa de otra amiga, porque ella la defendía y o sea, yo ya estaba harta de defenderla y ella seguía haciendo las mismas cosas Para mí fue muy difícil porque vamos a la misma clase, siempre salimos juntas y claro, estarla mirando todo el día pues no era fácil y no sé lloré muchas veces».

En general, en comparación con las co-étnicas, en las diadas interétnicas se reportan más emociones desagradables involucradas en el conflicto entre amigos, predominando la tristeza y el enojo. Los motivos pueden derivarse de desacuerdos, pero si hay algo que no perdonarían a sus amigos es la falta de lealtad.

Si nos planteamos que son diferentes culturas las que están interactuando y que están en una etapa donde se fortalece la identidad, tal vez su manera de reaccionar sea parte del proceso de adecuación de actitudes futuras y el encausar esto sería de gran ayuda para los adolescentes.

\section{Superación de conflictos en Amistades Co-étnicas e Inter-étnicas: Negociación y Alivio}

Tanto en diadas co-étnicas como inter-étnicas, los tipos de estrategias que utilizan para la resolución de un conflicto son similares. Primero intentan hablar de lo sucedido y solucionar los problemas y esto les provoca sensación de alivio, se sienten reconfortados porque las cosas van mejorando y eso les da tranquilidad y bienestar. Ejemplos de cómo superan los conflictos en diadas co-étnicas los encontramos en Dolors (14), catalana, que relata un conflicto con su amiga por llegar varias horas tarde a su casa para realizar un trabajo, expresó: "Le dije que lo sentía mucho, me sentía muy culpable. Luego que lo conversamos, me dijo que no volviera a llegar tarde, le pedí disculpas e hicimos el trabajo». En diadas inter-étnicas, los conflictos se solucionan de la misma manera, piden disculpas, como lo hizo Cristian (16) de Chile: "Con ella aprendí que es importante pedir perdón, aunque tú sepas que 

AMISTADES CO-ÉTNICAS E INTER-ÉTNICAS EN LA ADOLESCENCIA: DIFERENCIAS...

no es importante para ti, ¿sabes? Le pedí perdón, y ahora intento controlarme un poco más porque sé que no le gusta que haga este tipo de bromas».

Cuando los adolescentes relatan los sentimientos que les provoca solucionar sus dificultades, tanto en diadas co-étnicas como inter-étnicas, los asocian a la sensación de alivio, refieren que sienten que la relación está bien y que sigue su curso. Después de conversar con su amigo y arreglar las cosas, Mercè (14) catalana comentó: «Me sentí muy bien porque después de estar un día pensando que ya no volvería a hablarle porque nos habíamos enfadado, luego ves que sí lo hablas... que una amistad por una tontería no se puede acabar, porque cuando unas personas son amigas yo creo que no hay casi nada que pueda distanciar la relación así sin más». Sus palabras reflejan las respuestas de varios adolescentes que contestaron de forma similar a esta pregunta, existe un vínculo especial de amistad que no se puede perder por intercambios de opinión o pequeñas peleas y buscan la solución negociando, conversando, lo que a su vez puede ser motivo de conocimiento y de fortalecimiento de la relación, como expresa Jumina (14) de Marruecos: "Lo conversamos, lo solucionamos y es motivo de más confianza. Cuando las cosas se pueden hablar, engrandecen la relación de amistad».

Para ambos tipos de diadas las estrategias utilizadas en la resolución de sus diferencias es la negociación, conversan de lo sucedido para llegar a un acuerdo y esto en general trae sensaciones de alivio, se sienten reconfortados por mantener su relación intacta y poder continuar disfrutando de la compañía de su amigo.

\section{CONCLUSIONES}

Este estudio se orientó a identificar y comparar los valores que definen la calidad, el conflicto y sus formas de resolución en amistades inter-étnicas y co-étnicas.

En primer lugar, nuestros hallazgos dejan ver que tanto en diadas coétnicas como en inter-étnicas los adolescentes se sienten motivados para relatar episodios relativos a su amistad. Esta observación confirma que las amistades recíprocas son un contexto propicio para que los adolescentes exploren el efecto de sus comportamientos sobre ellos mismos y sobre los demás (Azmitia et al., 2005; Newman, et al., 2011; Rubin et al., 2008).

Más concretamente, los resultados indican que la calidad de las amistades en contextos escolares interculturales está condicionada principalmente por el nivel de confianza que logran establecer los amigos en ambos tipo de diadas, lo que hasta cierto punto es esperable porque es una expec- 
tativa básica de la amistad entre adolescentes (Linden-Andersen et al., 2008; Vaquera y Kao, 2008).

Sin embargo, se aprecian algunas diferencias cuando se comparan estos dos tipos de amistades. En las diadas integradas por amigos del mismo origen (co-étnicas) además de la confianza, los amigos valoran significativamente la compañía y las oportunidades para compartir diferentes actividades, ya sea dentro o fuera del Instituto. Probablemente esta preferencia responda a la similitud de intereses en gustos y aspiraciones, asociada a sus identidades culturales (Buhrmester, 1990; Bukowski, et al., 1994) y en cierta medida explica el «encapsulamiento étnico» que se ha observado en estudios sobre la composición de las amistades adolescentes (Alarcón, 2010; Álvarez et al., 2013; De Miguel y Carvajal, 2007; Hallinan y Williams, 1989; McGlothlin y Killen, 2006; Schneider et al., 2007). En cambio, en las diadas inter-étnicas (aparte de la confianza) los amigos atribuyen un significado especial a la ayuda que se genera en el contexto de su amistad, valoran poder dar y recibir apoyo, ya sea porque necesitan un consejo, porque están tristes y buscan consuelo o bien porque el apoyo que puede ofrecer el amigo de otra cultura favorece su inserción social y académica; en definitiva, les ayuda a integrarse y de esta manera se sienten partícipes de la sociedad de acogida (Newman, et al., 2011; Vaquera y Kao, 2012). Cuando la diada está conformada por un amigo de origen extranjero y uno de origen autóctono (inter-étnicas), este apoyo es especialmente valorado. Los adolescentes de origen extranjero destacan la ayuda que el amigo de la cultura de acogida (en este caso, Cataluña) le pueda brindar en acciones tan básicas como por ejemplo, el aprendizaje del idioma o bien puede ser la puerta de entrada para conocer y relacionarse con más jóvenes de esta cultura.

Por una parte, estos resultados también confirman los potenciales beneficios de las relaciones de amistad para el ajuste psicosocial (Álvarez et al., 2013; Bagwell y Schmidt, 2011; Wentzel et al., 2004). Por otra parte, es probable que el hecho de convivir con adolescentes de distintas culturas promueva mayor sensibilidad para aceptar, incluso para tolerar valores y costumbres diferentes a las propias (Azmitia et al., 2005; Noack y Rutland, 2012). Con ello, la relación de amistad se convierte realmente en un escenario ideal para aprender a regular las emociones y para desarrollar competencias sociales (Buhrmester, 1990).

En relación a los conflictos, y concordantes con los hallazgos de Burk y Laursen (2005) los adolescentes en su mayoría (independientemente de la composición étnica de la diada) intentan minimizarlos, dejando entrever que les da temor perder la amistad con este amigo cercano. En los dos tipos de diadas analizadas, encontramos que las discusiones/peleas ocurren cuando tienen desacuerdos y luego de discutir les queda una sensación de 

AMISTADES CO-ÉTNICAS E INTER-ÉTNICAS EN LA ADOLESCENCIA: DIFERENCIAS...

enfado, rabia o sienten el orgullo herido. Por el contrario, cuando hay pesar por haber discutido, se sienten tristes y desanimados por la ausencia del amigo.

En específico, los conflictos por desacuerdos de diadas inter-étnicas tienden a ser más profundos y significativos. Pueden ocurrir por algún problema entre la diada o bien generarse por discutir sobre la conducta que el amigo tiene frente a otras personas. Es recurrente también en las narrativas de los estudiantes de origen extranjero la molestia que sienten cuando se ríen de ellos o cuando los corrigen por no tener bien adquirido el idioma; se sienten ofendidos, avergonzados y heridos cuando los demás les hacen ver sus errores lingüísticos. Adicionalmente, este tipo de diadas considera la falta de lealtad del amigo como un motivo que pone en peligro la amistad. Dentro de los motivos más mencionados se encuentran el descubrir una mentira del amigo, el revelar algo íntimo o que el amigo hable de ellos a las espaldas.

No obstante, independientemente de la composición de la diada, los adolescentes entrevistados manifestaron voluntad expresa de querer superar los conflictos con sus amigos, similar a los resultados de otras investigaciones que han indagado al respecto (Bagwell y Schmidt, 2011; Rubin, Bukowski y Parker, 2006). Esta positiva actitud es especialmente importante para el mantenimiento de las relaciones de amistades interculturales (Pica-Smith, 2009; Serra, 2006). Los relatos de los adolescentes sugieren que cuando los amigos aceptan y valoran las diferencias étnicas, se favorece el establecimiento de relaciones de amistad inter-étnicas dentro del contexto escolar intercultural. De hecho, como muestran los resultados presentados, las amistades interculturales potencian la tolerancia y la búsqueda de acuerdos frente a las discrepancias en la relación, ocasionadas por malentendidos $\mathrm{o}$, tal vez, debido a insuficiente sensibilidad ante las diferencias que generan diversas perspectivas culturales. Es interesante que predomine la negociación para afrontar los conflictos y/o desacuerdos entre amigos de diverso origen. Este hecho sugiere que los adolescentes dominan estrategias efectivas para la resolución de conflictos interpersonales, con lo cual también se puede esperar que estas amistades tiendan a tener menos discrepancia y, consecuentemente, mayor calidad (Chong et al., 2013).

En términos generales, nuestros resultados avalan las conclusiones de los estudios consultados, tanto en relación a los aspectos que determinan la calidad de la amistad en la adolescencia como a su función en el ajuste psicosocial. En particular esta investigación ofrece indicios relativos a valores específicos que motivan las relaciones de amistad en contextos multiculturales que, en nuestra opinión, sugieren la conveniencia de promover las 
amistades inter-étnicas, sobre todo en sociedades con significativa presencia de población de origen extranjero como es el caso de España.

En concreto, en el ámbito escolar la relación de amistad intercultural puede constituir un contexto propicio para facilitar el ajuste y el bienestar de los adolescentes. Los centros de educación secundaria son un escenario excelente para fomentar actividades que conlleven compartir intereses y que comprometan la ayuda mutua y el trabajo en equipo. Durante su realización, es muy probable que se establezcan amistades de calidad, cuyo mantenimiento en el tiempo trasciende al escenario de origen, ofreciendo un nicho excepcional para el desarrollo psicológico en este período. Además, cuando hay compromiso relacional de amigos, hay buen clima escolar, lo que a su vez podría ayudar a mejorar la brecha académica existente hoy en día en las aulas, favorecer la obtención de mejores resultados de los estudiantes y, en definitiva, la deseada convivencia. 


\section{REFERENCIAS BIBLIOGRÁFICAS}

Alarcón, A. (Coord) (2010). Joves d origen immigrant a Catalunya. Necessitats $i$ demandes. Una aproximació sociològica. Institut Joventut. Generalitat de Catalunya. Departament d'Acció Social i ciutadania. Secretaria de Joventut.

Aparicio, R., Portes, A. y Haller, W. (2009). La 'Segunda generación' en Barcelona: Un estudio longitudinal. Informe de investigación. Recuperado de http://goo.gl/Q8CNos

Álvarez, I., Hernández, E. y Vall, B. (2013). Quins son els amics dels adolescents inmigrants a Catalunya? Quina funció fan en la seva adaptació a la cultura del l'escola? Recerca i immigracio V. Generalitat de Catalunya. Departament de Bienestar Social i Familia. Col.lecció Ciudadania i Inmigració, 9.

Álvarez, I., Schneider, B. \& Villalobos, C. (2015). School Adjustment and Friendship Quality of First- and Second-Generation Adolescent Immigrants to Spain as a Function of Acculturation. Journal of Adolescent Research, 1-28. doi:10.1177/0743558415592179.

Azmitia, M., Ittel, A. \& Radmacher, K. (2005). Narratives of friendship and self in adolescence. New Directions for Child and Adolescent Development, 107, 23-39. doi: 10.1002/cd.119.

Bagwell, C. \& Schmidt, M. (2011). Friendship in chilhood \& adolescence. New York: The Guilford Press.

Buhrmester, D. (1990). Intimacy of friendship, interpersonal competence, and adjustment during preadolescence and adolescence. Child Development, 61, 1101-1111.

Bukowski, W.M. (2004). Research on children's and adolescents friends- hips: four old and new questions that deserve our attention. International Society for the Study of Behavioural Development, 46(2), 7-10. Recuperado de http://goo.gl/JKu3gW

Bukowski, W.M. \& Hoza, B. (1989). Popularity and friendship: Issues in theory, measurement, and outcome. En T.J. Berndt \& G.W. Ladd (eds.), Peer relationships in child development (pp.15-45). New York: Wiley.

Bukowski, W.M., Hoza, B. \& Boivin, M. (1994). Measuring friendship quality during pre- and early adolescence: The development and psychometric properties of the friendship qualities scale. Journal of Social and Personal Relationships, 11(3), 471-484. doi: 10.1177/0265407594113011

Burk, W.J. \& Laursen B. (2005). Adolescent perceptions of friendship and their associations with individual adjustment. International Journal of Behavioral Development, 29(2), 156-164. doi: 10.1080/01650250444000342

Chong C. M., Holly R. \& Buhrmester D. (2013). The mediating role of interpersonal competence between adolescents empathy and friendship quality: a dyadic approach. Journal of Adolescence, 36, 191-200.

De Miguel, V. y Carvajal, C. (2007). Percepción de la inmigración y relaciones de amistad con los extranjeros en los institutos. Revista Migraciones, 22, 147-190.

Demir M. \& Urberg, K., (2004). Friendship and adjustment among adolescents. J. Experimental Child Psychology, 88, 68-82. doi: 10.1016/j. jecp.2004.02.006 

AMISTADES CO-ÉTNICAS E INTER-ÉTNICAS EN LA ADOLESCENCIA: DIFERENCIAS..

Feddes, A. R., Noack, P. \& Rutland, A. (2009). Direct and extended friendship effects on minority and majority children's interethnic attitudes: A longitudinal study. Child Development, 80(2), 377-390. doi: 10.1111/j.14678624.2009.01266.x

Generalitat de Catalunya (2012). Butlletí secretaría per a la Inmigració. La inmigració en xifres, 13, http://goo.gl/ CxpxeD

Giró, J. (2011). Las amistades y el ocio de los adolescentes, hijos de la inmigración. Papers, 96(1), 77-95.

Hallinan, M.T. \& Williams, R. (1989). Interracial friendship choices in secondary schools. American Sociological Review, 54, 67-78.

INE (2012). Revisión del Padrón municipal 2011. Datos a nivel nacional, comunidad autónoma y provincia. Recuperado de http://goo.gl/2MlJ2H

Linden-Andersen, S., Markiewicz, D. \& Doyle, A. (2008). Perceived similarity among adolescent friends: The role of reciprocity, friendship quality, and gender. Journal of Early Adolescence, 17, 617-637. doi: $10.1177 / 0272431608324372$

McGlothlin, H. \& Killen, M. (2006). Intergroup attitudes of European American children attending ethnically homogeneous schools. Child Development, 77(5), 1375-1386. doi: 10.1111/j.1467-8624.2006.00941.x

Munniksma, A., Juvonen, J. (2012). Cross-ethnic friendships and sense of social-emotional safety in a multiethnic middle school: An exploratory study. Merrill-Palmenr Quarterly, 58(4) 489-506. doi: 10.1353/mpq.2012.0023

Newman, K. J., Erdley, C.A. \& Marshall, K. C. (2011). Peer acceptance and friendship as predictors of early ado- lescents' adjustment across the middle school transition. Merrill-Palmer Quarterly, 57(3), 215-243.

Pica-Smith, C. (2009). Children speak about interethnic and interracial friendships in the classroom. Multicultural Education, 17(1), 38-47.

Rubin, K.H., Bukowski, W.W. \& Parker, J.G. (2006). Peer interactions, relationships, and group. En W. Damon, N. Eisenberg \& R. M. Lerner (Ed.), Handbook of child psychology. (Sixth Edition) (pp. 619-699). NY: Wiley.

Schneider B., Dixon, K. \& Udvari, S. (2007). Friendships of early adolescents in Toronto and Montreal closeness and competition in the inter-ethnic and co-ethnic. The Journal of Early Adolescence, 27, 115-137. doi: $10.1177 / 0272431606294822$.

Schneider, B., Malik, Y. \& Udvari, S. (2012). Cultural encapsulation of children's friendships: A manifestation of prejudice or pride. Journal of Psychology and Education, 7(1), 2343.

Sharabany, R. \& Schneider, B. (2004). On the study of friendship in childhood and adolescence: A view from that bridge(s). International society for the study of behavioural development, 46(2), 1-5.

Serra, C. (2006). Diversitat, racisme $i$ violència. Conflictes a l' educació secundaria. Barcelona: Eumo.

Smith, A. \& Schneider, B. (2000). The inter-ethnic friendships of adolescent students: a Canadian study. International Journal of Intercultural Relations, $24,247-258$.

Vaquera, E. \& Kao, G. (2008). Do you like me as much as I like you? Friendship reciprocity and its effects on 
school outcomes among adolescents. Social Science Research, 37, 55-72. doi: 10.1111/j.1467-8624.2012.01791.x

Vaquera, E. \& Kao, G. (2012). Educational achievement of immigrant adolescents in Spain: Do gender and region of origin matter? Child Development,
83(5), 1560-1576. doi: 10.1016/j.ssresearch.2006.11.002

Wentzel, K., McNamara, B. \& Caldwell, A. (2004). Friendships in middle school: Influences on motivation and school adjustment. Journal of Educational Psychology, 96(2), 195-203. doi:10.1037/0022-0663.96.2.195 


\section{PERFIL ACADÉMICO Y PROFESIONAL DE LAS AUTORAS}

Cecilia Francisca Villalobos Carrasco. Doctora en Psicología de la Educación por la Universidad de Barcelona. Su tesis de investigación se centró principalmente en las relaciones de amistad que establecen los adolescentes en contextos educativos de diversidad cultural con presencia significativa de inmigrantes. En específico, el estudio se focalizó en las amistades de los adolescentes de origen inmigrante que establecen relaciones de amistad diádica en el contexto de la escuela.

Ibis Marlene Álvarez Valdivia. Doctora en Psicología, profesora de la Facultad de Psicología de la Universitat Autònoma de Barcelona y consultora de la Universitat Oberta de Catalunya. Integra el Seminario Interuniversitario de Investigación en Estrategias de Enseñanza y Aprendizaje (SINTE). Sus investigaciones se centran en el desarrollo de la personalidad en contextos sociales multiculturales y en estrategias de evaluación y enseñanzaaprendizaje en la universidad.

Elizabeth Vaquera. Profesora Asociada (con tenure) del departamento de Sociología de la University of South Florida. Doctora en Sociología por la University of Pennsylvania. Sus ámbitos de investigación se focalizan en áreas de la sociología de la Educación, de la adolescencia y de la juventud, además realiza estudios en inmigración e hispanos. Es autora de diversas publicaciones en libros y revistas y co-autora del libro Education and Immigration (2013) entre otros.

Dirección de las autoras: Cecilia Villalobos

Universidad de Barcelona

Passeig de la Vall d'Hebron, 171

08035 Barcelona

E-mail: cecilia.villalobos.ca@gmail.com

Ibis Álvarez

Universitat Autònoma de Barcelona

Departament: Bàsica, Evolutiva i de

l'Educació

Edifici B, Campus de la UAB

08193 Bellaterra (Cerdanyola del Vallès)

E-mail: ibismarlene.alvarez@uab.cat

Elizabeth Vaquera

University of South Florida

4202 E. Fowler Avenue

Tampa, FL 33620 
Department of Sociology, CPR 107

E-mail: evaquera@usf.edu

Fecha Recepción del Artículo: 22. Febrero. 2014

Fecha modificación Artículo: 11. Julio. 2014

Fecha Aceptación del Artículo: 21. Julio. 2014

Fecha Revisión para Publicación: 06. Julio. 2016 